\title{
Contrast Enhancement with Dual Energy CT for the Assessment of Atherosclerosis
}

\author{
Stefan C. Saur ${ }^{1}$, Hatem Alkadhi ${ }^{2}$, Luca Regazzoni ${ }^{1}$, Simon Eugster ${ }^{1}$, \\ Gábor Székely ${ }^{1}$, Philippe Cattin ${ }^{1,3}$ \\ ${ }^{1}$ Computer Vision Laboratory, ETH Zurich, Switzerland \\ ${ }^{2}$ Institute of Diagnostic Radiology, University Hospital Zurich, Switzerland \\ ${ }^{3}$ Medical Image Analysis Center, University of Basel, Switzerland \\ saur@vision.ee.ethz.ch
}

\begin{abstract}
A drawback of the commonly used single source computed tomography systems (CT) is that different materials might show very similar attenuation at any selected radiation energy. However, the assessment of atherosclerosis requires good differentiation between vessel lumen, calcium, adipose, and surrounding tissue. Dual energy CT (DECT) simultaneously measures attenuations at two energies and therefore can improve the differentiation to some extent. A tissue cancelation and enhancement algorithm for dual energy data was already proposed in 1981 and evaluated on experimental settings with a stationary X-ray source. For this study, we adapted this algorithm for DECT and propose its usage as a pre-processing step for the assessment of atherosclerosis. On clinical DECT patient data and with fixed parameters we could show a simultaneous contrast enhancement between $8 \%$ and $67 \%$ among all targeted tissues.
\end{abstract}

\section{Introduction}

Each material has a characteristic X-ray attenuation depending on its atomic number, density and the incident energy of the X-ray beam. Single source computed tomography systems (CT) characterize tissues with only one value such that different materials might show very similar attenuation. For the assessment of atherosclerosis, however, a precise differentiation between different tissues is needed: Calcified hard plaques are detected by differentiating calcium from the vessel lumen (blood/iodine), whereas for non-calcified soft plaques, vessel lumen, adipose and surrounding tissue must be distinguished.

A dual energy CT system (DECT) has two X-ray sources and therefore allows to simultaneously measure the X-ray attenuation at low and high energy levels such that two attenuation values are present for each volume element (voxel). This additional dimension can be used for better material discrimination. Dual energy X-ray acquisitions have been discussed and evaluated on basic phantoms since the early 1980s $[1,2,3]$ until the first commercial DECT system was introduced 2005 [4]. On this DECT system, Eusemann et al. [5] evaluated different 
blending techniques to combine the low and high energy images into one improved single image whereas Johnson et al. [6] used unpublished algorithms to estimate the iodine content of each voxel from the low and high energy images.

In 1981, Lehmann et al. [1] proposed a tissue cancelation algorithm by decomposing the low and high energy images into two basis material images and combining them afterwards into a single projection image. Depending on a single parameter, the algorithm cancels out the contrast between two materials to enhance a third one, or it enhances the contrast between materials - both demonstrated on experimental settings with a stationary X-ray source. We adapted this algorithm for clinical DECT and propose its usage as a pre-processing step for the assessment of atherosclerosis. We applied the method on clinical dualenergy (DE) data and measured the contrast enhancement and signal to noise ratio between several tissues of interest. With one fixed parameter setting we could simultaneously enhance the contrast between all targeted tissues.

\section{Methods}

The principle of the tissue cancelation algorithm by Lehmann et al. [1] is summarized in Fig. 1 and described below in detail. First, the original low and high energy images are decomposed into two basis material images to map the density distribution of two arbitrarily chosen basis materials. Then, the two bases are combined in a cancelation step into a single projection image.

The two attenuation coefficients $\mu$ (low) and $\mu$ (high) obtained by DECT for each voxel are decomposed into a basis pair $\rho_{A}$ and $\rho_{B}$ of two chosen basis materials $\mathrm{A}$ and $\mathrm{B}$ by

$$
\begin{aligned}
& \rho_{A}=\frac{1}{\alpha}\left[\mu(\text { high }) \mu_{B}(\text { low })-\mu(\text { low }) \mu_{B}(\text { high })\right] \\
& \rho_{B}=\frac{1}{\alpha}\left[\mu(\text { low }) \mu_{A}(\text { high })-\mu(\text { high }) \mu_{A}(\text { low })\right]
\end{aligned}
$$

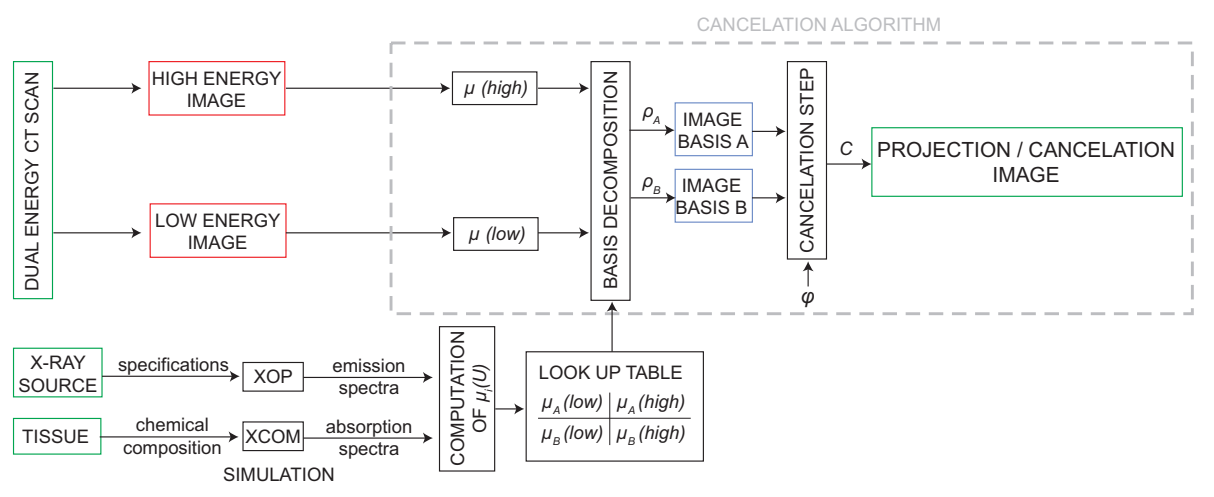

Fig. 1. Workflow of the tissue cancelation algorithm proposed by Lehmann et al. [1] 
Table 1. For each possible material combination of lumen, tissue, adipose, and calcium, we determined the cancelation angle $\varphi_{\max }$ that maximized the mean contrast enhancement factor $(\overline{\mathrm{CEF}})$ measured between the cancelation image and the $140 \mathrm{kV}$ acquisition over all data sets.

\begin{tabular}{lcccc}
\hline & lumen & tissue & adipose & calcium \\
\hline $\max \overline{\mathrm{CEF}}\left(\varphi_{\max }\right)$ lumen & - & $1.58(0.81)$ & $2.82(0.84)$ & $1.30(0.81)$ \\
$\max \overline{\mathrm{CEF}}\left(\varphi_{\max }\right)$ tissue & $1.58(0.81)$ & - & $1.16(0.80)$ & $1.38(0.81)$ \\
$\max \overline{\mathrm{CEF}}\left(\varphi_{\max }\right)$ adipose & $2.82(0.84)$ & $1.16(0.80)$ & - & $1.48(0.81)$ \\
$\max \overline{\mathrm{CEF}}\left(\varphi_{\max }\right)$ calcium & $1.30(0.81)$ & $1.38(0.81)$ & $1.48(0.81)$ & - \\
\hline
\end{tabular}

with $\alpha=\mu_{B}($ low $) \mu_{A}($ high $)-\mu_{B}$ (high) $\mu_{A}($ low $)$. The attenuation coefficient $\mu_{i}(U)$ of the basis material $i$ at the tube voltage $U$ is calculated by $\mu_{i}(U)=$ $\frac{\int_{0}^{\infty} N_{U}(E) \mu_{i}(E) d E}{\int_{0}^{\infty} N_{U}(E) d E}$ where $N_{U}(E)$ is the photon energy distribution at tube voltage $U$ and $\mu_{i}(E)$ is the attenuation spectrum for material $i$. We used [7] to compute $N_{U}(E)$ of a Tungsten X-ray tube $\left(5 \mathrm{~mm} \mathrm{Al}\right.$ filter) and for $\mu_{i}(E)$ we used a photon cross sections database [8]. For this study, we considered all possible basis material pair combinations between iodine, calcium, water, polymethyl methacrylate, adipose and blood.

The projection, or cancelation, image is computed by $C=\rho_{A} \sin (\varphi)+$ $\rho_{B} \cos (\varphi)$ with $\varphi$, the cancelation angle, as a parameter.

For the assessment of atherosclerosis, a good contrast between vessel lumen, soft tissue, adipose, and calcium is desired. Therefore, we manually selected a region $R_{i}$ within a data set for each material $i(i=1 . .4)$. The mean intensity $\bar{I}_{i}$ and standard deviation $\sigma_{i}$ are computed within each $R_{i}$ for every data set. The cancelation angle $\varphi$ is varied between $-\pi$ and $+\pi$ with a step of 0.01 . For each selected $\varphi$, the contrast enhancement factor (CEF) between material $i$ and $j$ is computed as

$$
\mathrm{CEF}_{i j}=\frac{\left|\bar{I}_{c_{i}}-\bar{I}_{c_{j}}\right|}{\bar{I}_{c_{i}}+\bar{I}_{c_{j}}} / \frac{\left|\bar{I}_{h_{i}}-\bar{I}_{h_{j}}\right|}{\bar{I}_{h_{i}}+\bar{I}_{h_{j}}},
$$

where CEF represents the enhancement ratio of the contrast in the cancelation image (index $c$ ) and the original high energy image (index $h$ ). Hence, a CEF $>1$ should be achieved. Further, the signal to noise ratio $S N R_{i}=\bar{I}_{i} / \sigma_{i}$ is measured for each material $i$ in the low and high energy, and cancelation image, respectively.

The cancelation angle is only dependent on the materials to be enhanced [1] such that we expected different optimal cancelation angles for the various targeted tissues which, however, should be universally applicable for all patient images. We evaluated this by measuring the CEF and SNR in dependence on $\varphi$. Further, we determined the optimal angles maximizing the contrast between two targeted tissues. 
Table 2. We measured the mean ( \pm std $)$ contrast enhancement factor $(\overline{\mathrm{CEF}})$ for the cancelation image $(\varphi=0.79)$ against the high energy $140 \mathrm{kV}$ acquisition for each possible material combination. Further, the mean $( \pm$ std) signal to noise ratio $(\overline{S N R})$ in the cancelation, the $80 \mathrm{kV}$, and the $140 \mathrm{kV}$ images was measured.

\begin{tabular}{lcccc}
\hline & lumen & tissue & adipose & calcium \\
$\overline{\overline{\mathrm{SNR}} \text { cancelation }}$ & $61.6( \pm 28.2)$ & $39.6( \pm 17.8)$ & $60.9( \pm 23.5)$ & $11.3( \pm 1.6)$ \\
$\overline{\mathrm{SNR}} 140 \mathrm{kV}$ & $51.4( \pm 11.7)$ & $37.8( \pm 10.9)$ & $56.7( \pm 15.5)$ & $14.7( \pm 6.3)$ \\
$\overline{\mathrm{SNR}}$ 80kV & $42.9( \pm 22.2)$ & $27.3( \pm 14.2)$ & $40.4( \pm 18.3)$ & $9.5( \pm 0.7)$ \\
$\overline{\mathrm{CEF}}$ lumen & - & $1.27( \pm 0.06)$ & $1.67( \pm 0.34)$ & $1.16( \pm 0.03)$ \\
$\overline{\mathrm{CEF}}$ tissue & $1.27( \pm 0.06)$ & - & $1.08( \pm 0.08)$ & $1.19( \pm 0.03)$ \\
$\overline{\mathrm{CEF}}$ adipose & $1.67( \pm 0.34)$ & $1.08( \pm 0.08)$ & - & $1.23( \pm 0.04)$ \\
$\overline{\mathrm{CEF}}$ calcium & $1.16( \pm 0.03)$ & $1.19( \pm 0.03)$ & $1.23( \pm 0.04)$ & - \\
\hline
\end{tabular}

\section{Results}

The presented method was evaluated on five abdominal DE scans showing both lipid and calcified plaques in the descending aorta. The data sets were acquired on a Siemens Definition DECT scanner (Siemens Medical Solutions, Forchheim, Germany). The study was approved by the local ethics committee. A clinical abdominal DE protocol was used with a tube voltage of $80 \mathrm{kV}$ for the low and $140 \mathrm{kV}$ for the high energy image, respectively. The in-plane resolution and slice spacing ranged between $0.68-0.87 \mathrm{~mm}$ with a slice thickness of $2 \mathrm{~mm}$.

In each patient, a region for lumen and tissue (both $7 \times 7 \times 9$ voxels), for adipose $(5 \times 5 \times 1$ voxels $)$, and for calcium $(3 \times 3 \times 1$ voxels $)$ was selected by an experienced reader. Among all basis material combinations, the pair calciumadipose showed the best results in terms of magnitude and robustness for the intended contrast enhancement such that this basis pair was used for this study.

Figure 3(a) exemplarily shows the CEF between adipose and calcium in dependence of $\varphi$ for all data sets. Our initial expectation regarding patient independent characteristics could be justified. The mean $\overline{\mathrm{CEF}}$ and mean $\overline{\mathrm{SNR}}$ over all data sets were computed and for each possible tissue combination, we determined an optimal cancelation angle $\varphi_{\max }$ that maximized $\overline{\mathrm{CEF}}$ (Table 1 ).

As all $\varphi_{\max }$ were very similar, we decided to determine one single optimal angle $\varphi^{*}$ that simultaneously enhances the contrast among all targeted tissues. Based on one data set, we chose the optimal angle $\varphi^{*}=0.79$ that maximized the CEF among all tissue combinations but also showed a good SNR for each material (Fig. 3(b)). A higher CEF would have been possible with a slightly larger $\varphi$ but that would have resulted in a worse SNR. The optimal angle $\varphi^{*}$ was then fixed and applied on all data sets. The contrast between all targeted tissues could be simultaneously enhanced between $8 \%$ and $67 \%$ (Table 2). Except for calcium, the SNR in the cancelation image was also higher for the materials than the SNR measured in the $80 \mathrm{kV}$ and $140 \mathrm{kV}$ data sets, respectively. 
Fig. 2. (a) Contrast enhancement factor (CEF) between adipose and calcium in dependence on the cancelation angle $\varphi$ for all data sets. The selected $\varphi^{*}$ (dashed) simultaneously enhanced the contrast among all targeted tissues in the cancelation image compared to the $140 \mathrm{kV}$ image - exemplarily shown in (b) and (c).
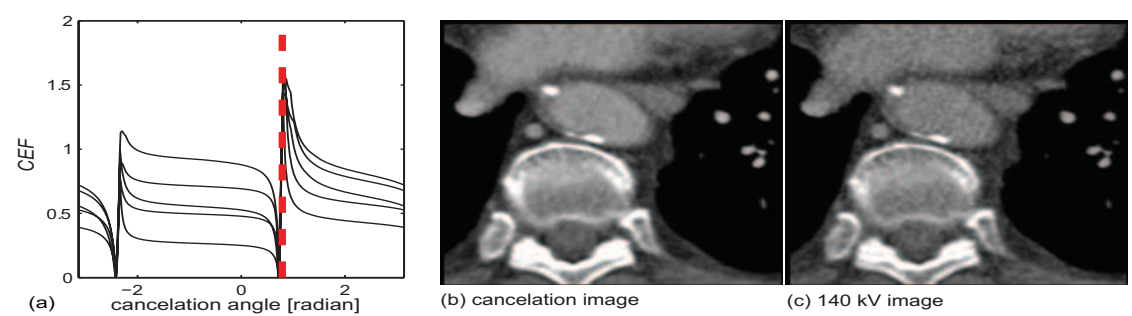

\section{Discussion and conclusion}

This study evaluated for the first time the performance of a tissue cancelation algorithm on real DECT patient data for contrast enhancement. We propose to use this procedure as a pre-processing step for the assessment of atherosclerosis to improve the robustness of both the manual reading and intensity-based detection algorithms. We could show by measuring the contrast enhancement between targeted tissues that there is one fixed, patient-independent optimal angle for the assessment of atherosclerosis as it enhances the contrast between all targeted tissues. Future work will concentrate on incorporating this contrast enhancement into radiological workstations and into algorithms for automatic detection and quantification of atherosclerosis.

Acknowledgement. This work has been supported by the CO-ME/NCCR research network of the Swiss National Science Foundation.

\section{References}

1. Lehmann LA, Alvarez RE, Macovski A, et al. Generalized image combinations in dual KVP digital radiography. Med Phys. 1981;8(5):659-667.

2. Marziani M, Taibi A, Tuffanelli A, et al. Dual-energy tissue cancellation in mammography with quasi-monochromatic x-rays. Phys Med Biol. 2002;47(2):305.

3. Taibi A, Fabbri S, Baldelli P, et al. Dual-energy imaging in full-field digital mammography: A phantom study. Phys Med Biol. 2003;48(13):1945-1956.

4. Flohr T, McCollough C, Bruder H, et al. First performance evaluation of a dualsource CT (DSCT) system. Eur Radiol. 2006;16(2):256-268.

5. Eusemann C, Holmes III DR, Schmidt B, et al. Dual energy CT: How to best blend both energies in one fused image? Procs SPIE. 2008;6918(1):691803-8.

6. Johnson T, Krauß B, Sedlmair M, et al. Material differentiation by dual energy CT: Initial experience. Eur Radiol. 2007;17(6):1510-1517.

7. del Rio MS, Dejus RJ. XOP: Recent developments. Procs SPIE. 1998;3448(1):340345.

8. Berger MJ, Hubbel JH. XCOM: Photon Cross Sections Database. National Institute of Standards and Technology; 1998. 